\title{
RASTREAMENTO DOS CASOS DE MALÁRIA DO ESTADO DO RIO DE JANEIRO NO PERÍODO DE 2007 A 2017
}

\section{TRACKING MALARIA CASES IN THE STATE OF RIO DE JANEIRO FROM 2007 TO 2017}

\author{
Camila Silva Cota ${ }^{1}$, Romulo da Silva Viana ${ }^{2}$, Diego da Silva Sales ${ }^{2}$, Camila Henriques Nunes ${ }^{2}$, Karla Rangel \\ Ribeiro², Camila Mendonca Romero Sales ${ }^{2}$ \\ ${ }^{1}$ Graduada em Engenharia Ambiental, Instituto Federal de Educação, Ciência e Tecnologia Fluminense - IF \\ Fluminense \\ ${ }^{2}$ Docente do Instituto Federal de Educação, Ciência e Tecnologia Fluminense - IF Fluminense
}

Autor para correspondência: E-mail: karla.ribeiro@iff.edu.br

Av. Souza Mota, 350 - Parque Fundão, Campos dos Goytacazes - RJ, 28060-010 Telefone: 022-99818-3707

\section{RESUMO}

A malária é um problema mundial de saúde pública que vem tendo um desenvolvimento significativo nos últimos tempos. O maior número de casos se concentra na região Amazônica, entretanto há uma carência de estudo sobre a doença nas outras regiões, como é o caso do estado do Rio de Janeiro. O principal objetivo é observar, através de cálculos de prevalência, as regiões e as cidades do estado do Rio de Janeiro mais afetadas. Os dados utilizados durante a pesquisa para a obtenção dos resultados foram adquiridos por meio do Banco de Dados do Sistema Único de Saúde (DATASUS). Com os valores de prevalência calculados, foram construídos gráficos distintos que possibilitaram a análise do desenvolvimento de malária nas regiões e cidades do estado. As regiões que se destacaram com maior prevalência da doença foram: Serrana, Norte e Metropolitana, juntamente às cidades de Santa Maria Madalena, Macaé e Rio de Janeiro respectivamente. Pode-se dizer que o maior desenvolvimento de malária nas áreas citadas está diretamente relacionado com as características ambientais da região como: vegetação, clima, saneamento e temperatura, além do número populacional e fluxo de pessoas. Para a minimização da malária nas regiões, determinadas medidas poderiam ser realizadas, como por exemplo, melhora no saneamento básico com foco no tratamento de esgoto, melhoria nas condições de habitação, conscientização da população juntamente à visitas domiciliares constantes por agentes de saúde.

Palavras-chave: Malária. Rio de Janeiro. Prevalência

\section{ABSTRACT}

Malaria is a worldwide public health problem that has been experiencing significant development in recent times. The largest number of cases is concentrated in the Amazon Region, however there is a lack of studies on the disease in other regions, as is the case in the Rio de Janeiro State. The main objective is to observe, through prevalence calculations, the most affected regions and cities in the state of Rio de Janeiro. The data used during the research to obtain the results were acquired through the "Banco de Dados do Sistema Único de Saúde" (DATASUS). With the calculated prevalence values, different graphs were constructed that made it possible to analyze the development of malaria in the regions and cities of the state. The regions that stood out with the highest prevalence of the disease were: Serrana, Norte and Metropolitana, together with the cities of Santa Maria Madalena, Macaé and Rio de Janeiro respectively. It can be said that the greatest development of malaria in the aforementioned areas is directly related to the environmental characteristics of the region, such as: vegetation, climate, sanitation and temperature, in addition to the population number and the flow of people. To minimize malaria in the regions, certain measures could be taken, such as, for example, improvement in basic sanitation with a focus on sewage treatment, improvement in housing conditions, awareness of the population along with constant home visits by health agents.

Keywords: Malaria. Rio de Janeiro. Prevalence 


\section{INTRODUÇÃO}

Desde a antiguidade a malária está presente em meio à população. Levando em consideração uma análise anual, por volta de 300 milhões de pessoas em todo o mundo acabam contraindo o vírus da malária. Dentro desta estimativa, 1,5 a 2 milhões de pessoas não sobrevivem (CAMARGO, 2003).

A doença ainda é considerada um problema de saúde pública mundial mesmo com todo o avanço realizado pela Organização Mundial da Saúde (OMS) a fim de conseguir seu combate até finais dos anos de 1970. Um dado alarmante é que a malária causa mais óbitos que a Síndrome da Imunodeficiência Adquirida (AIDS) levando em consideração valores absolutos, sendo a África o país mais afetado (FRANÇA; SANTOS; FIGUEROAVILLAR, 2008).

Sua maior incidência se dá em áreas tropicais e também subtropicais do planeta, sendo assim, a África, o Sudeste Asiático e a Região Amazônica da América do Sul são as áreas mais afetadas (REINERS et al., 2010).

No Brasil, observa-se uma maior incidência da doença na região Norte. Seu desenvolvimento está diretamente relacionado com as condições insatisfatórias presentes em determinadas regiões, principalmente de trabalho e habitação, assim como a exploração manual de minérios e o aumento da saída de pessoas que antes habitavam na zona rural e passam a ocupar as periferias das grandes cidades da região amazônica. Além disso, a região Norte do país possui altas temperaturas que, somadas a má infraestrutura sanitária e ao bioma com clima adequado para a proliferação dos mosquitos transmissores, faz com que a região tenha um grande número de casos da doença (MOREIRA, 2017).

Apesar da alta incidência da malária na região norte do Brasil, a causa de morte pela doença na região extra-amazônica é por volta de 80 vezes maior do que na região amazônica, que é definida como endêmica, concentrando $99,8 \%$ dos casos de todo o país. Como o Rio de Janeiro é uma região com altos índices de casos de dengue, há muitas vezes o tardio diagnóstico e tratamento dos pacientes com malária devido à similaridade dos sintomas iniciais de ambas, o que pode confundir os profissionais de saúde (COSTA et al., 2010).

Pode-se perceber que há um aumento no número de casos no Estado do Rio de Janeiro. Nesse sentido, o objetivo deste estudo é analisar qualiquantitativamente os casos de malária no estado do Rio de Janeiro, de 2007 a 2017, e rastrear as regiões e cidades que possuem o maior número de casos de acordo com o cálculo de prevalência. Com isso, espera-se que esse trabalho possa contribuir para o entendimento do desenvolvimento da doença e seu perfil no estado do Rio de janeiro ajudando em futuras estratégias de vigilância em saúde na região.

\section{METODOLOGIA}

Este trabalho é baseado no estudo epidemiológico com a finalidade de descrever a ocorrência e desenvolvimento da malária no estado do Rio de Janeiro relacionando-a com as características espaciais, temporais, climáticas e de população acometida.

Reuniu-se informações bibliográficas e dados quantitativos na plataforma DATASUS obtidos por meio do Sistema de informação TABNET do Banco de Dados do Sistema Único de Saúde para a obtenção tanto do número de casos confirmados em cada cidade que compõe o estado do Rio de Janeiro assim como a estimativa populacional de cada uma nos anos de 2007 a 2017. Em seguida, foram analisadas quais as regiões e cidades que apresentaram uma maior prevalência de casos desta doença. Para tal análise utilizou-se da fórmula

Prevalência $=$ № de casos confirmados da doença em um determinado período * 100.000 / População durante o mesmo período.

Os gráficos e as tabelas foram construídos através do programa de computador MS Office Excel 2010.

\section{RESULTADOSE DISCUSSÃO}

\subsection{NÚMERO DE CASOS, POPULAÇÃO E PREVALÊNCIA DAS CIDADES}

Com base no Sistema de informação TABNET do DATASUS foram identificados 800 casos de malária confirmados em todo o estado, de 2007 a 2017. As cidades do Rio de Janeiro, Macaé e Nova Friburgo foram as que apresentaram um maior número de casos. As cidades que apresentaram as maiores prevalência são Macaé, Santa Maria Madalena e Rio de Janeiro, com prevalência total de 51.704 ou $48,86 \%$ do total.

\subsection{ANALISE DA PREVALÊNCIA NAS REGIÕES DO ESTADO DO RIO DE JANEIRO}


Com base nas divisões das Regiões do estado do Rio de Janeiro conforme a Lei $n^{\circ} 1.227 / 87$ (CEPERJ, 2019b) foi elaborado um estudo mais aprofundado de cada região apresentado através dos gráficos de 1 a 8.

No gráfico 1, referente às Baixadas Litorâneas, Rio das Ostras se destaca com uma prevalência de 2,07 casos por 100.000 habitantes no ano de 2009 enquanto Araruama apresenta uma maior prevalência no ano de 2008 com 0,93. Por fim, diferentemente, tem-se a cidade de Cabo Frio com uma maior prevalência em 2014 no valor de 0,49 .

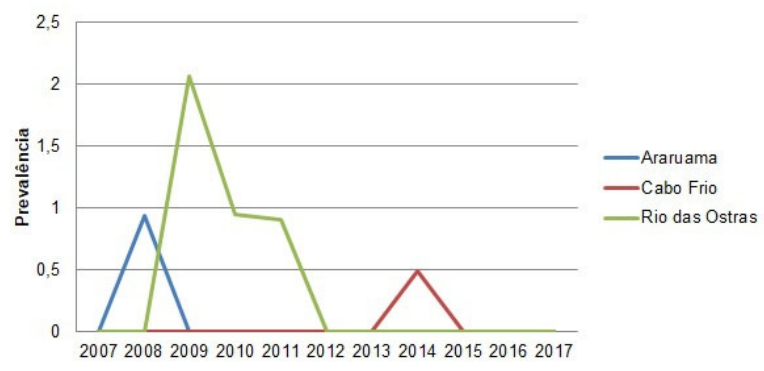

Gráfico 1 - Prevalência de malária (casos por 100.000 habitantes) das cidades da região das Baixadas Litorâneas do estado do Rio de Janeiro no período de 2007 a 2017

Fonte: Autoria Própria.

A região Norte representada no Gráfico 2 evidencia que a cidade de Macaé se sobressai com os valores de prevalência. $\mathrm{O}$ ano de maior pico de prevalência em Macaé é em 2010, quando apresentou prevalência de 5,80 casos por 100.000 habitantes. Já em Campos dos Goytacazes, o ano de maior prevalência foi em 2014 com um valor de 0,62 , ou seja, um valor cerca de 9 vezes menor. Além disso, Macaé registrou números de casos em todos os anos, exceto em 2017. Campos dos Goytacazes apresentou casos somente em 2009, 2011 e 2014. A região Norte está entre as que apresentaram maiores prevalências.

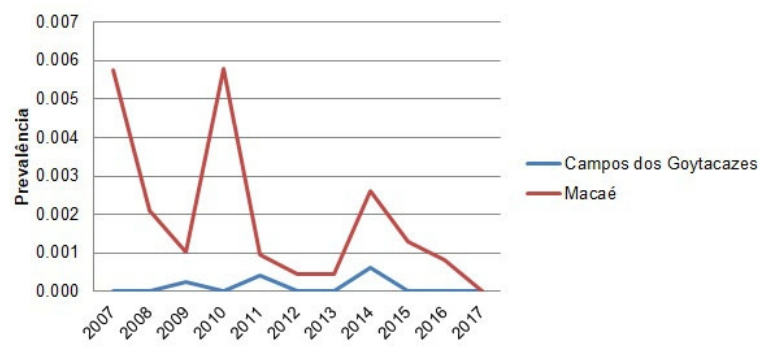

Gráfico 2 - Prevalência de malária (casos por 100.000 habitantes) das cidades da região Norte do estado do Rio de Janeiro no período de 2007 a 2017.

Fonte: Autoria Própria.
O Gráfico 3 mostra a cidade de Vassouras, a única com casos confirmados de malária na região Centro-Sul. Possuiu apenas casos no ano de 2012 que gerou uma prevalência de 2,87 por 100.000 habitantes. Neste ano foi registrado apenas 1 caso, não sendo uma região que se destacou dentre as demais analisadas.

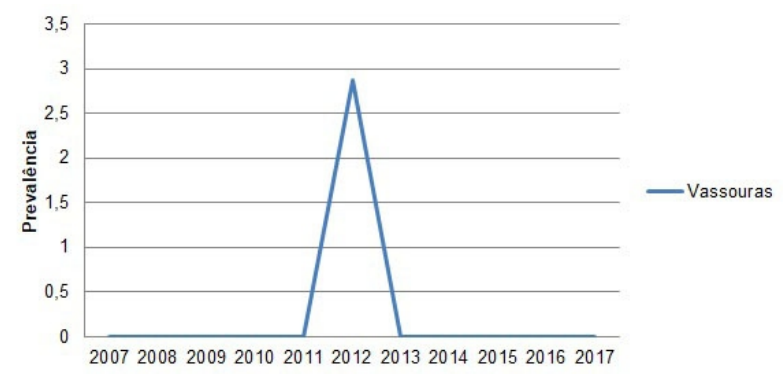

Gráfico 3 - Prevalência de malária (casos por 100.000 habitantes) das cidades da região Centro-Sul do estado do Rio de Janeiro no período de 2007 a 2017.

Fonte: Autoria Própria.

A Região Metropolitana, como pode-se perceber no Gráfico 4, é a que possui o maior número de cidades com casos registrados de malária. A região está entre as que mais possuem prevalência entre todas analisadas. A cidade do Rio de Janeiro é a que se destaca apresentando mais casos no ano de 2012 e com casos registrados em todos os anos analisados exceto 2017. Em seguida, aparece a cidade de Cachoeiras de Macacu com casos nos anos de 2009, 2010 e 2014 e Guapimirim em 2007 com quase 2,5 de prevalência e 2015 com cerca de 1,7 .

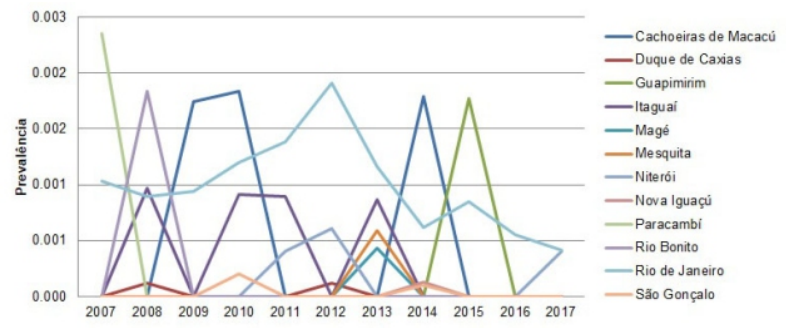

Gráfico 4 - Prevalência de malária (casos por 100.000 habitantes) das cidades da região Metropolitana do estado do Rio de Janeiro no período de 2007 a 2017.

Fonte: Autoria Própria.

Por meio do Gráfico 5 é possível perceber que a Região Noroeste apresentou duas cidades com casos confirmados de malária. Ambas tiveram casos apenas no ano de 2007. Porciúncula apresentou uma prevalência de 5,83 , um pouco maior em relação à Itaperuna que teve um valor de 3,23. A região não está dentro das que apresentaram maiores quantidades de 
prevalências.

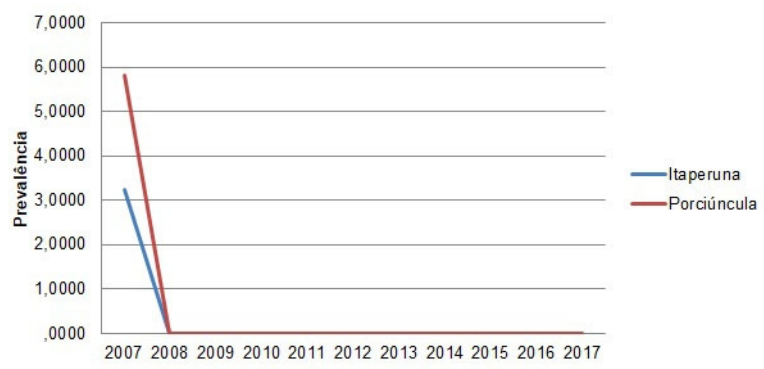

Gráfico 5 - Prevalência de malária (casos por 100.000 habitantes) das cidades da região Noroeste do estado do Rio de Janeiro no período de 2007 a 2017.

Fonte: Autoria Própria.

A Região Serrana, apresentada no Gráfico 6, possui 4 cidades com casos confirmados, destacando-se Santa Maria Madalena com picos de prevalência nos anos de 2012 e 2014, com 9,71 e 9,75 respectivamente. Nova Friburgo apresentou casos durante 6 dos 11 anos analisados com um total de prevalência igual a 8,21. Cordeiro apresentou apenas casos no ano de 2015 com prevalência de 4,75. A cidade de Petrópolis é a que chama mais atenção já que o número de casos aumentou no ano de 2017, sendo a sua maior prevalência. Uma hipótese para tal evidência seria a crise do lixo ocorrida no começo do ano de 2017 na cidade (FERNANDES, 2018).

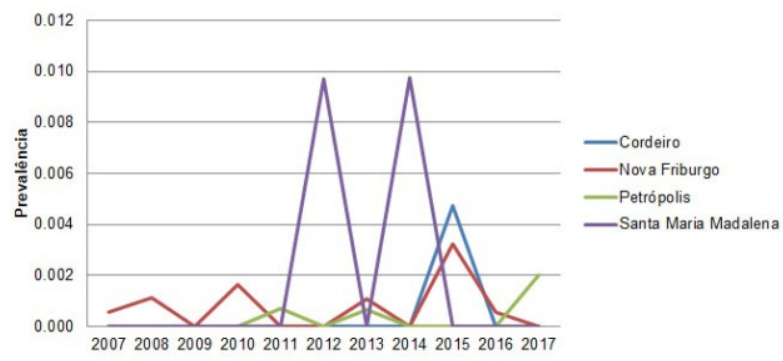

Gráfico 6 - Prevalência de malária (casos por 100.000 habitantes) das cidades da região Serrana do estado do Rio de Janeiro no período de 2007 a 2017.

Fonte: Autoria Própria.

Pode-se perceber no Gráfico 7 que apenas a cidade de Angra dos Reis registrou casos de malária em toda a Região de Costa Verde. Os anos de 2012 e 2017 são os que possuem casos com prevalência de 0,57 e 0,52 respectivamente, possuindo uma soma de 1.60. A região foi a que apresentou a menor soma de prevalência entre as demais, apenas $1 \%$ do total.

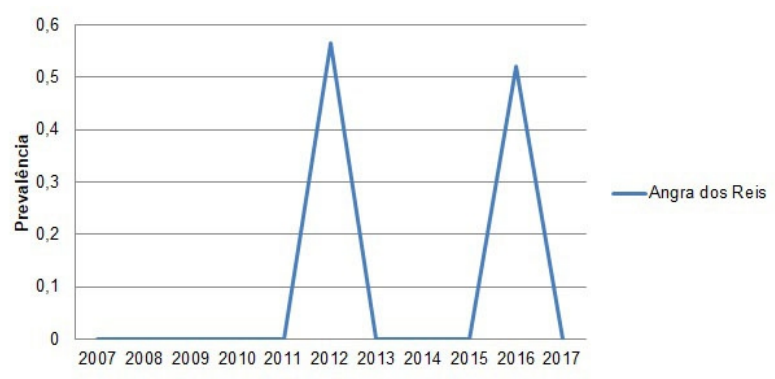

Gráfico 7 - Prevalência de malária (casos por 100.000 habitantes) das cidades da região Costa Verde do estado do Rio de Janeiro no período de 2007 a 2017.

Fonte: Autoria Própria.

Três cidades apresentaram casos na região Médio Paraíba (Gráfico 8): Barra do Piraí, Resende e Volta Redonda. A região não aparece entre as que apresentaram grandes números de prevalência apresentando um total de 3,449.

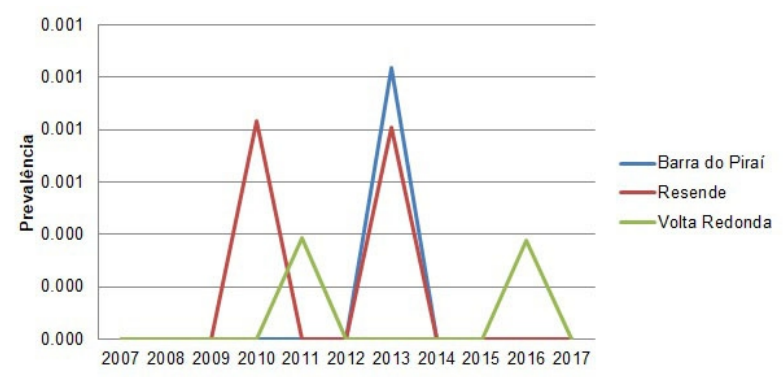

Gráfico 8 - Prevalência de malária (casos por 100.000 habitantes) das cidades da região Médio Paraíba do estado do Rio de Janeiro no período de 2007 a 2017.

Fonte: Autoria Própria.

Após a descrição de todas as regiões, através do Gráfico 9 é possível observar a porcentagem das prevalências calculadas para cada região. Dessa forma pode-se afirmar que as cidades que apresentaram maiores porcentagens de prevalência em relação à malária entre 2007 e 2017 foram a Região Serrana com 33\%, a Região Metropolitana com $26 \%$ e a Região Norte Fluminense com $21 \%$.

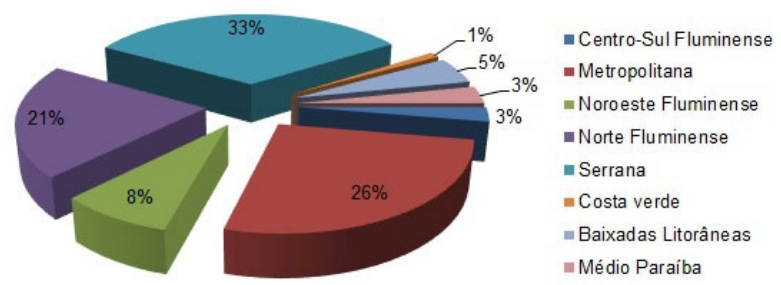

Gráfico 9 - Porcentagem de Prevalência de malária (casos por 100.000 habitantes) em cada região do estado do Rio de Janeiro no período de 2007 a 2017.

Fonte: Autoria Própria. 


\subsection{ANÁLISE DAS REGIÕES COM MAIOR PORCENTAGEM DE PREVALÊNCIA}

\subsubsection{Região Serrana}

A região Serrana Fluminense situa-se na porção central do estado do Rio de Janeiro. Seu clima é caracterizado como Tropical de Altitude. As chuvas são presentes durante todo o ano, entretanto se tornam mais concentradas no período do verão (DOURADO; ARRAES; SILVA, 2013).

Possui uma cobertura de abastecimento de água em torno de $76,83 \%$, de coleta de esgoto de $53,83 \%$ e de tratamento de esgoto de $28,03 \%$, ou seja, em média mais de $70 \%$ de todo o esgoto da região Serrana do Rio de Janeiro não possui tratamento adequado (FIRJAN, 2017).

Entre as cidades que compõem a região, quatro delas apresentaram casos notificados de malária nos anos estudados: Cordeiro, Nova Friburgo, Petrópolis e Santa Maria Madalena. A grande ocorrência de casos pode ser por conta do seu clima úmido, onde há intensas chuvas no verão. A temperatura mais elevada no verão com o aumento de chuvas, bem como deslizamentos que geram acúmulo de resíduos ajuda na reprodução do mosquito transmissor, o que justifica a região ser líder no valor de prevalências (DANTAS et al., 2005).

A cidade com maior prevalência na região foi a de Santa Maria Madalena. O clima regional é visto como inverno seco e verão chuvoso com temperaturas em torno de 22 o C. Na encosta Atlântica e também no alto da Serra do Imbé há grande quantidade de chuvas o ano inteiro. A cidade faz parte da faz parte da Bacia Hidrográfica do Imbé (DRZ GEOTECNOLOGIA E CONSULTORIA, 2012). A cidade apresentou a maior prevalência em 2012 e 2014, superando todas as outras cidades. O clima úmido e relativamente quente no verão com altas quantidades de chuvas favorecem a proliferação do mosquito transmissor das doenças, facilitado também pela boa quantidade de corpos hídricos na região.

A cidade de Petrópolis registrou, em 2017, 6 casos, com prevalência de 2,01, a maior entre todas as cidades neste ano, provavelmente devido a uma crise do lixo ocorrida.

\subsubsection{Região Metropolitana}

A região Metropolitana é hoje o segundo maior polo de concentração demográfica do Brasil. Com isso, possui um grande volume de fluxos de pessoas e de atividades além de uma elevada taxa de urbanização (LUCENA et al., 2012).

A ação antrópica sobre a natureza levou a geração de problemas ambientais: inundações, poluições atmosféricas e da água, extremos térmicos. Além disso, é observado na região metropolitana o fenômeno das ilhas de calor, originadas principalmente das transformações na mudança de cobertura da superfície (LUCENA et al., 2012).

A justificativa do grande número de casos na região poderia ser, primeiramente, por ser uma região altamente urbanizada e com um grande número de pessoas e, por conta disso, mais casos são registrados. O intenso fluxo de pessoas entrando e saindo da região principalmente por ser uma área com grande fluxo de atividades que aliada a uma baixa coleta e baixo tratamento de esgoto facilitam a ocorrência da doença.

A cidade do Rio de Janeiro é vista como a segunda maior marcha urbana de todo país. Todo ano é recebido na cidade mais de 1,4 milhão de turistas de outros países e cerca de 3 milhões de visitantes. Sua pluviosidade total aumenta cerca de $1.200 \mathrm{~mm}$ ao ano, ocorrendo com mais intensidade entre os meses de novembro a janeiro. No verão a temperatura média chega a ser de $36,9 \circ \mathrm{C}$, sendo muitas vezes a capital mais quente do país (DERECZYNSKI; OLIVEIRA; MACHADO, 2009). O valor de sua prevalência chegou a 10,96, registrando 685 casos durante todos os anos analisados.

\subsubsection{Região Norte Fluminense}

Atualmente a região Norte Fluminense é composta por 9 municípios. Havia uma economia considerável no setor sucroalcooleiro e, com o desenvolvimento do petróleo, deu-se uma nova dinâmica para a economia da região, especialmente na cidade de Macaé gerando muitos empregos e aumentando o fluxo de pessoas (SILVA, 2005).

No que se diz respeito à vegetação, percebe-se que uma grande parte das terras é ocupada por pastagens. A pluviosidade média anual na região é de 1177,6 mm, sendo dezembro o mês mais chuvoso. A temperatura média anual varia em torno dos $22 \circ$ C. Apresenta altas quantidades de corpos hídricos (BURLA et al., 2012). O abastecimento de água na região abrange cerca de $85,75 \%$ do local, a coleta de esgoto é feita em $39,15 \%$ da região e seu tratamento em $11,04 \%$ (FIRJAN, 2017). 
A cidade que aparece com mais destaque de prevalência na região é Macaé. Esta possui uma elevada concentração de pessoas no centro urbano, apresentado uma taxa de urbanização de $98,1 \%$ (SILVA; FARIA, 2011). Apenas $30,1 \%$ do esgoto é coletado em Macaé (FIRJAN, 2017). A cidade apresentou 37 casos durante os 11 anos a prevalência total foi de 21,28 , sendo a maior dentre todas as cidades que compõem o estado. As cidades de Macaé e Santa Maria Madalena possuem determinadas semelhanças, o que pode justificar esse grande número de casos de malária em regiões próximas geograficamente. $06^{\circ}$ distrito de Macaé, o Sana, pode ter contribuição para o aumento de número de casos. A região se localiza na parte serrana de Macaé e é conhecida pelas cachoeiras e áreas de acampamento. Na área predominam superfícies de Mata Atlântica (JERONYMO, 2012).

\subsubsection{Comparação e discussão entre as cidades} de Macaé, Santa Maria Madalena e Rio de Janeiro

Como visto, Macaé, Santa Maria Madalena

e Rio de Janeiro foram as cidades com maiores destaques nas Regiões Norte, Serrana e Metropolitana respectivamente. No Gráfico 10 é possível analisar o comportamento e desenvolvimento da prevalência de malária de cada uma dessas cidades no decorrer dos anos 2007 a 2017.

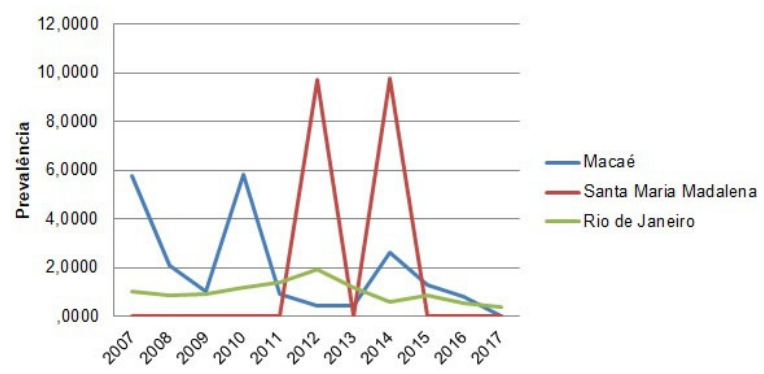

Gráfico 10 - Prevalência de malária (casos por 100.000 habitantes) nas cidades de Macaé, Santa Maria Madalena e Rio de Janeiro no período de 2007 a 2017.

Fonte: Autoria Própria.

É possível perceber que a cidade de Santa Maria Madalena tem destaque no que se diz respeito aos maiores valores de prevalência em relação a Macaé e Rio de Janeiro. Entretanto, estas últimas cidades possuem maior número de casos confirmados, enquanto Santa Maria Madalena possuem casos apenas em 2012 e 2014. Também é possível perceber que Macaé se sobressai quando comparada à cidade do Rio de Janeiro principalmente nos primeiros anos analisados.

As cidades citadas anteriormente são destacadas no mapa do estado do Rio de Janeiro apresentado na Figura 1.

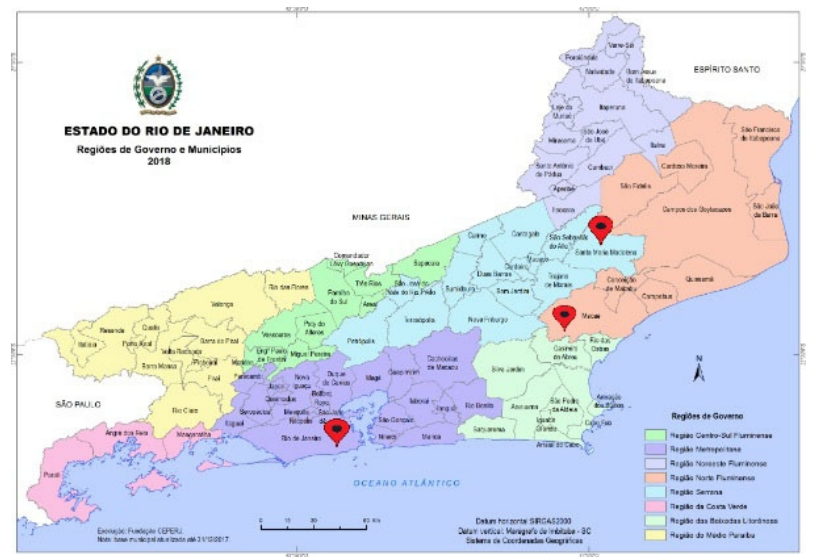

Figura 1 - Mapa do estado do Rio de Janeiro com a identificação das cidades com maiores somas de prevalência de malária no estado do Rio de Janeiro.

Fonte: Adaptado de CEPERJ (2019c).

Neste mapa nota-se a proximidade das cidades de Santa Maria Madalena e Macaé, embora se encontrem em regiões distintas. Além da proximidade, as duas cidades possuem semelhança no que se diz respeito à vegetação. Santa Maria Madalena e o distrito do Sana, pertencente a Macaé, são áreas com intensa vegetação típica da Mata Atlântica, que aliada às cachoeiras favorecem a formação de microclimas e o desenvolvimento do mosquito transmissor. Devido às suas características naturais, recebem muitos visitantes, estando então mais propícias a adquirir a malária. Também é importante ressaltar que todo o estado do Rio de Janeiro possui mais de 100 Estruturas hidráulicas, entre elas barragens, estando cerca de 6 dessas estruturas na cidade de Santa Maria Madalena (DIAS, 2016).

\section{CONCLUSÃO}

A malária ainda é considerada um problema de saúde pública mundial. É uma doença com maior incidência em áreas tropicais, com a Região Amazônica, porém são registrados casos significativos nas regiões extra-amazônicas, como o Estado do Rio de Janeiro. Por não ser uma doença tão comum no estado, ainda é difícil realizar seu diagnóstico, já que apresenta sintomas semelhantes a outras doenças. 
O número de casos de malária está diretamente relacionado com determinadas características que variam de região. Em relação à temperatura, o mosquito possui um melhor desenvolvimento e reprodução entre 20 e $33^{\circ} \mathrm{C}$ e com bastante pluviosidade.

Por conta desse fator, regiões bem próximas a rios, lagos e cachoeiras também tendem a possuir um maior número de casos de malária, principalmente se estivem próximas às áreas de floresta mais densas.

Com isso, pessoas que vivem próximos a locais com tais características estão mais propícias a adquirirem a doença, assim como trabalhadores rurais que exercem suas atividades diretamente no campo e por longo período de tempo, já que os mosquitos podem utilizar da vegetação da pastagem como abrigo. Áreas com moradias sem saneamento básico adequado também são mais propícias à doença.

Pode-se perceber que a Região Serrana destacou-se com a cidade de Santa Maria Madalena. A região como um todo possui temperaturas mais amenas, porém no verão com o seu leve aumento aliado às chuvas constantes favorecem a reprodução do mosquito. Além disso, também possuem áreas com quantidades significativas de vegetação da Mata Atlântica.

A Região Norte também apresentou número significativo de prevalência, sobretudo a cidade de Macaé, que possui o distrito do Sana, área turística sobretudo pela presença de cachoeiras e muita vegetação característica de Mata Atlântica. A cidade de Macaé em si também possui um alto fluxo de pessoas devido aos empregos gerados pelas indústrias de petróleo, aumentando também a probabilidade de malária na região.

A região Metropolitana também teve destaque com a cidade do Rio de Janeiro, com maior número de habitantes, além de ser altamente urbanizada e com intensos fluxos de pessoas, além de possuir determinadas áreas sem condições adequadas de habitação. Tais fatores podem ser justificativos para os números de casos encontrados.

É importante ressaltar que a falta de saneamento básico ainda é um problema enfrentado no Brasil, principalmente no que se diz respeito a tratamento de esgoto. Tal fator colabora para o aumento de diferentes doenças além da malária. Com maiores investimentos nessa área, muitas doenças seriam diminuídas, assim como melhorias na saúde da população em geral.

Outras ações que ajudariam no combate à malária seriam melhorar as condições de habitação e também a conscientização da população por meio de divulgações de informações de medidas preventivas que ajudariam no combate ao mosquito, como por exemplo, evitar o acúmulo de água. Visitações domiciliares por agente de saúde pública também é um fator positivo no combate à doença. Aos vetores adultos e larvas, o combate seria por meio do borrifamento de inseticidas e larvicidas respectivamente, porém é importante ressaltar que prevenir o problema é mais fácil se comparado a combatê-lo depois.

\section{REFERÊNCIAS BIBLIOGRÁFICAS}

Burla RS., Silva Neto R., Werneck LG, Maciel CP., Silva RA, Pessanha HM, Oliveira VPS. Análise das condicionantes socioeconômicas e ambientais para a implantação da silvicultura nas regiões Norte e Noroeste Fluminense. Boletim do Observatório Ambiental Alberto Ribeiro Lamego, Campos dos Goytacazes, 2012, 6(1): 85-113

Camargo, EP. Malária, maleita, paludismo. Ciência e Cultura, 2003, 55(1): 26-29.

CEPERJ. Fundação CEPERJ - Centro Estadual de Estatísticas Pesquisas e Formação de Servidores Públicos do Rio de Janeiro. Disponível em: <http://www.ceperj.rj.gov.br/ceep/info_territorios/divis_regional.html>. Acesso em: 25 jan. $2019 a$.

Costa AP, Bressan CS, Pedro RS, Valls-De-Souza R, Silva S, Souza PR, Guaraldo L, Ferreira-Da-Cruz MF, Daniel-Ribeiro CT, Brasil P. Diagnóstico tardio de malária em área endêmica de dengue na extra-Amazônia Brasileira: experiência recente de uma unidade sentinela no estado do Rio de Janeiro. Rev. Soc. Bras. Med. Trop., Uberaba, 2010, 43(5): 571-574.

Dantas, ME. et al. Diagnóstico geoambiental do estado do Rio de Janeiro. Repositório Institucional de Geociências (RiGeo). Anais... In: OFICINA INTERNACIONAL DE ORDENAMENTO TERRITORIAL MINEIRO, CIENCIA Y TECNOLOGIA PARA EL DESAROLLO COOPERACION IBERO-AMERICANA. Rio de Janeiro - RJ: 2005. Disponível em:

<http://rigeo.cprm.gov.br/jspui/handle/doc/644>

Dereczynski CP; Oliveira JS; Machado CO. Climatologia da precipitação no município do Rio de Janeiro. Revista Brasileira de Meteorologia, 2009, 24(1): 24-38. 
Dias, FS. Segurança de Barragens no Estado do Rio de Janeiro. Publicado por: Comitê para Integração da Bacia Hidrográfica do Rio Paraíba do Sul (CEIVAP), , 2016. Disponível em: <http://www.ceivap.org.br/barragem/apresentacao-inea-01-062016.pdf>. Acesso em: 31 jan. 2019

Dourado F, Arraes TC, Silva MF. O Megadesastre da Região Serrana do Rio de Janeiro - as Causas do Evento, os Mecanismos dos Movimentos de Massa e a Distribuição Espacial dos Investimentos de Reconstrução no Pós-Desastre. Anuário do Instituto de Geociências - UFRJ, 2013, 35_2(1): 43-54.

DRZ GEOTECNOLOGIA E CONSULTORIA. Plano Regional de Saneamento com Base Municipalizada nas Modalidades Água, Esgoto e Drenagem Urbana, 2012. Disponível em: <http://cbhbaixoparaiba.org.br/saneamento/campos/CaracterizacaoMunicipal.pdf>. Acesso em: 17 ago. 2018

Fernandes P. Restropectiva da cidade de Petrópolis. Disponível em: <http://www.diariodepetropolis.com.br/integra/retrospectiva-143846>. Acesso em: 25 jan. 2019.

FIRJAN. Saneamento no estado do Rio de Janeiro. Publicado por: Federação das Indústrias do estado do Rio de Janeiro (FIRJAN), , 2017. Disponível em:

<http://www.firjan.com.br/lumis/portal/file/fileDownload.jsp?fileld=2C908A8F5FFF1747016007D245D060D1>. Acesso em: 31 jan. 2019

França TCC, Santos MG, Figueroa-Villar JD. Malária: aspectos históricos e quimioterapia. Química Nova, 2008, 31(5): 1271-1278.

Jeronymo CAL. Identificação de Áreas Prioritárias, com uso de Geoprocessamento, para conservação e recuperação da área de preservação ambental do Sana, Macaé-RJ. 2012.

Lucena AJ, Rotunno Filho OC, Peres LF, França JRA. A evolução da ilha de calor na região metropolitana do Rio de Janeiro. Revista Geonorte, 2012, 2(5): 8-21.

Moreira DL. Aspectos epidemiológicos da malária no Rio Grande do Sul. 2017.

Reiners AAO, Azevedo RCSouza, Ricci HA, Souza TG. Adesão e reações de usuários ao tratamento da malária: implicações para a educação em saúde. Texto contexto - enferm. 2010, 19(3): 536-544.

Silva SRA, Faria TJP. O mapa da migração em Macaé: impactos da industrialização no processo de urbanização. Anais do Seminário Nacional da Pós-Graduação em Ciências Sociais - UFES, 2011, 1(1).

Silva, LC. Reconfiguração do Norte Fluminense a partir dos anos 70: a chegada do petróleo e suas consequencias na dinâmica de crescimento regional. 2005. 